\title{
Population-Based Multilevel Models to Estimate the Management Strategies for Acute Myocardial Infarction in Older Adults with Dementia
}

Yunfei Li (iD)

Akira Babazono'

Aziz Jamal $\mathbb{D}^{1,2}$

Ning Liu (iD ${ }^{3}$

Reiko Yamao'

'Department of Health Care Administration \& Management, Graduate School of Medical Sciences, Kyushu University, Fukuoka, Japan; ${ }^{2}$ Health Administration Program, Faculty of Business \& Management, Universiti Teknologi Mara, Selangor, Malaysia; ${ }^{3}$ Department of Preventive Medicine and Community Health, University of Occupational and Environmental Health, Kitakyushu, Japan
Correspondence: Yunfei Li; Aziz Jamal Department of Health Care Administration \& Management, Graduate School of Medical Sciences, Kyushu University, Fukuoka, Japan

Tel +8I-80-8088-6969; +8I-80-8497-5375 Email yunfei.li90@gmail.com; azizabduls. jamal@gmail.com
Background: Acute myocardial infarction (AMI) management strategies, involving treatment and post-care, are much more difficult for patients with dementia. This study investigated the factors influencing the use of invasive procedures and long-term care in the management strategies for AMI patients with dementia and the factors associated with these patients' survival.

Methods: This multilevel study combined information from two databases, namely laterstage elderly healthcare insurance and long-term care insurance claims, from 2013 to 2019. Of 214,963 individuals with dementia, we identified 13,593 patients with AMI. The primary outcomes were the use of invasive procedures for treatment and long-term care for post-care management. Survival outcomes were also measured over a 6-year period, adjusting for individual- and regional-level characteristics in multilevel models.

Results: A total of 1954 (14.38\%) individuals received an invasive procedure during treatment, and $7850(87.18 \%)$ used long-term care for post-care management after AMI. After multivariate adjustment, patients aged $\geq 85$ years and women were less likely to receive invasive procedures and more likely to use long-term care. Patients undergoing invasive procedures had a lower use of long-term care. Better survival outcome was significantly associated with invasive management and long-term care, regardless of the type of care.

Conclusion: Age and sex determine the use of invasive procedures and long-term care after AMI among patients with dementia. AMI patients with dementia receiving invasive procedures and long-term care had better survival outcomes.

Keywords: acute myocardial infarction, dementia, invasive procedure, long-term care, older adult, multilevel analysis

\section{Introduction}

Acute myocardial infarction (AMI) is a leading cause of death among older adults. $^{1,2}$ AMI management strategies, which involve both treatment and postcare, are much more difficult for patients with dementia, which is an age-related syndrome that can result in reduced quality of life. ${ }^{3}$ Dementia is a syndrome in which there is deterioration in memory, thinking, behaviour and the ability to perform everyday activities. Because of its progressive, incurable and life-limiting characteristics, people with dementia require positive treatment and care strategies. Very often, care in a variety of clinical settings and long-term care focuses on promoting well-being and maintaining optimal functioning. 
Invasive procedures - including percutaneous coronary intervention (PCI) and coronary artery bypass grafting (CABG) - have been demonstrated to be effective treatment strategies, contributing to improved survival outcomes for AMI; ${ }^{4-6}$ however, a lower use of invasive procedures has been reported among older patients with dementia. $^{7,8}$ Treatment strategies are more conservative for older AMI patients with dementia mainly because of comorbidities and a lack of guidelines and instructions. Dementia becomes a barrier to invasive management strategy for AMI patients. ${ }^{9}$

Long-term care is recommended for patients with dementia and for those needing care after discharge, such as after AMI. ${ }^{10-13}$ Long-term care involves multiple services to help people live as independently and safely as possible, for example during post-discharge recuperation or for those having difficulties performing everyday activities. ${ }^{14,15}$ In Japan, individuals can receive long-term care in each secondary medical area (SMA; the unit for care service provision governed by each prefecture under Japan's Medical Service Law). ${ }^{16}$ Some previous research has reported that long-term care provision is associated with improved quality of life and improved survival outcomes among older patients with dementia and AMI. ${ }^{12,14}$

Clinicians, specialists and individuals face difficulties in making treatment and post-care management strategy decisions for AMI patients with dementia. ${ }^{17,18}$ Therefore, we conducted a 6-year study of AMI patients with dementia in Japan. The main objectives of this study were to investigate 1) the factors influencing the use of invasive coronary management procedures in the treatment strategy and the use of long-term care in the post-care management strategy and 2) whether invasive procedures and long-term care were associated with better survival outcomes for patients.

\section{Methods}

\section{Participants}

This was a population-based multilevel study. We obtained data from the Health Insurance Association in Fukuoka Prefecture, Japan. In this study, we extracted data from the latter-stage elderly healthcare insurance (LEHI) database and the long-term care insurance (LTCI) database. The LEHI database contains information on individuals enrolled in insurance plans designed specifically for residents aged $\geq 75$ years and those aged 65-74 years with a specified disability. Information on sex, birth date, region of residence, and diagnostic and treatment procedures are available in the database. The LTCI database includes claims information and data on sex, birth date and longterm care utilisation for adults aged $\geq 65$ years. The data were extracted for April 1, 2013-March 31, 2019.

In this study, we identified 214,963 patients with dementia records in the LEHI database. We then selected those who had AMI hospitalisation records as the main diagnosis occurring after the dementia records. International Classification of Diseases 10th Revision (ICD-10) codes were used to identify dementia and AMI status. For dementia, Alzheimer's disease was defined by codes F00 and G30, vascular dementia by F01 and other dementia by F03 and F04. Mixed dementia was defined by more than two of the codes mentioned above or by ICD-10 codes of F02, F05.1, F10.7, F12.7, G31.0 and G31.1. For AMI, ST-Elevation Myocardial Infarction (STEMI) was defined by codes I21.0-I21.3 and non-STEMI by codes of I21.4 and I21.9. We ultimately identified 13,593 eligible patients. The type of diagnosis procedure combination (DPC) in the database (whether DPC or not) was used to distinguish patients with emergency acute conditions. DPC was introduced in 2002 to contain health expenditures and to improve the quality of care in Japanese care facilities to cover most acute in-patient care.

\section{Comorbidities and Drugs}

We used ICD-10-coded data to examine nine comorbidities, following a previous study: ${ }^{19}$ shock, diabetes mellitus, heart failure, cardiac dysrhythmia, cerebrovascular disease, pulmonary oedema, acute renal disease, chronic renal disease and cancer. In this study, we included "shock" as one of the comorbidities. Shock in this context includes cardiogenic shock, hypovolaemic shock, septic shock and unspecified shock due to failure of peripheral circulation. Each comorbidity was assigned a score of one point if present, and the total comorbidity score was calculated by summing these points.

We extracted patients' information on drugs taken at least once up to 1 year before and 1 year after the date of AMI diagnosis. We extracted this information using the codes in the Kyoto Encyclopedia of Genes and Genomes, a set of codes applied uniformly throughout Japan. Specifically, we extracted data on antithrombotic agents, statins, beta blockers, calcium channel blockers, angiotensin-converting enzyme inhibitors (ACEs)/angiotensin receptor blockers (ARBs), vasodilators, diuretics, antidiabetics, 
lipid-lowering drugs, cholinesterase inhibitors, antipsychotics, anxiolytics, hypnotics and sedatives.

\section{Home-Visit Treatment and Long-Term Care}

Home-visit treatment targets patients with difficulty going to the hospital who wish to receive medical treatment at home. Information on the use of home-visit treatment was extracted from the LEHI database. For patients using longterm care, the types of care are home-visit care, residential facility care and outpatient facility care. Although homevisit care and residential facility care are seemingly related to more severe conditions and a lower level of quality of life, the selection of the types of long-term care is determined by both the patient's need and the physician's decision. $^{20}$ The Japanese government also encourages older adults with chronic diseases, especially those with dementia to use long-term care services, and many older patients prefer to receive residential facility care or homevisit care because of its convenience. ${ }^{20,21}$ Therefore, we assume that patients utilise long-term care services not only because of severe conditions of diseases but also because of their individual needs. Information on the use of long-term care was extracted from the LTHI database. We identified the use of home-visit treatment and longterm care up to 3 months after the date of AMI. We combined information from the LTHI and LEHI databases using patient's identification number, a shared unique identifier.

The databases we used cover more than 600,000 insured older people annually, and the penetration rate of insurance coverage is as high as $98.6 \%$ based on our previous studies. $^{22}$ The diagnoses (including dementia and AMI) that were identified using ICD-10 codes from claim databases, showed an acceptable reliability as demonstrated in previous studies, thus could be applied to studies analysing healthcare data from the Japanese insurance and long-term care claims database. ${ }^{23,24}$ A previous study investigated the standardization of disease names and classifications according to ICD-10 codes showed a high degree of utility whereby only $1.9 \%$ of the data failed to converge. ${ }^{25}$ In addition, Japanese Health Insurance Claims Review \& Reimbursement Services is responsible for the quality control of computeradministered claims databases. Therefore, we have reason to believe that the missing data-if present-would be minimal, and the quality of our database can be ensured.
The data extraction was performed using SQL Server 2014. This study was approved by Kyushu University's Institutional Review Board (Clinical Bioethics Committee of the Graduate School of Medical Sciences, Kyushu University). Because we used anonymised claims insurance data, informed consent was not required.

\section{Explanatory Variables}

Individual-level characteristics included sex, age, comorbidities, drugs taken, invasive management status, DPC status, use of home-visit treatment and type of long-term care. We categorised age into the following groups: 65-74 years, 75-84 years, 85-94 years and $\geq 95$ years. Invasive procedures were defined as the use of PCI or CABG during the hospitalisation for AMI. Long-term care was classified into four groups: home-visit care, residential facility care, outpatient facility care and no long-term care.

The data description and the sources of the SMA-level characteristics are listed in Table S1. We first examined the correlations of each variable. The percentage of people aged over 75 years was excluded as a covariate because it was highly correlated with population density $(r=0.793$, 95\% confidence interval [CI]: 0.787-0.799) and psychiatric bed density ( $r=0.696,95 \%$ CI: $0.687-0.704)$. Chronic care bed density was excluded as a covariate because it was highly collinear with dementia rate $(r=0.758,95 \%$ CI: 0.751-0.765). Psychiatrist density was highly collinear with bed density in general clinics $(r=0.719,95 \% \mathrm{CI}$ : $0.710-0.727)$ and with general bed density $(r=0.733$, 95\% CI: 0.726-0.741). Because psychiatrist density was a more important predictor for this study, we excluded the other two variables as covariates. Long-term care facility density was excluded as a covariate because of high collinearity with nursing home staff density $(r=0.932,95 \%$ CI: 0.929-0.934). When possible, SMA-level characteristics were coded based on 2014-2015 estimates.

\section{Analytical Approach}

We present descriptive statistics as mean \pm standard deviation (SD), median and interquartile range (IQR) or frequency (n, \%). We conducted stratified analyses by sex, type of AMI, invasive management procedure, type of dementia and type of long-term care. To compare characteristics between groups, we used the independent samples $t$-test or one-way analysis of variance for normally distributed continuous variables, the Mann-Whitney $U$-test or the Kruskal-Wallis test for continuous variables with 
skewed distributions, and the chi-square test or Fisher's exact test for categorical variables.

Common statistical analyses (e.g., simple linear regression, ANOVA-like statistics) might not be able to quantify random variations when the data have a hierarchical or clustered structure. ${ }^{26,27}$ Therefore, in this situation, analyses using multilevel model is preferred. We fitted two-level multilevel models to evaluate the influence of covariates on patient outcomes, setting patients as level 1 and SMAs as level 2. Multilevel logistic models were used to examine which factors determined the use of invasive management and long-term care. We estimated multilevel logistic models in Stata 15 using the xtmelogit command. First, we performed Model 1 to examine the use of invasive management procedures, with age, sex, number of comorbidities, total number of drugs and DPC status as predictors. Then, in Model 2, we estimated the use of long-term care and its effects, with age, sex, number of comorbidities, total number of drugs, DPC status, invasive management and home-visit treatment status as predictors. We estimated odds ratios (ORs) with $95 \%$ confidence intervals (CIs). In both models, we calculated the SMA-level variance $\left(\delta^{2}\right)$, the intraclass correlation coefficient (ICC) for similarity within SMAs ${ }^{27,28}$ and the median odds ratio (MOR) for variance between SMAs. ${ }^{29}$

Subsequently, we estimated multilevel survival models specifying a Weibull distribution to compare survival status between patients with and without invasive management and among patients with different types of long-term care. We used right-censoring for the study data. The patients were followed from the time of AMI until death or the end of follow-up on March 31, 2019, whichever came first. We estimated the multilevel survival models in Stata 15 using the mestreg command and specified a Weibull distribution. ${ }^{30}$ Hazard ratios (HRs) with $95 \%$ CIs were computed. We incorporated the individual-level characteristics in Model 3. In Model 4, we added the SMA-level characteristics to Model 3 to estimate the survival outcome and effects for AMI patients with dementia. SMA-level differences in outcomes were adjusted by including a random effect for SMA in both models. All statistical analyses for this study were performed using Stata 15 (StataCorp, College Station, TX, USA).

\section{Results}

\section{Descriptive Analysis}

In total, 13,593 patients with dementia diagnosed with AMI were identified in 13 SMAs (incidence rate: 63.2/
1000 population). The mean age of patients at the time diagnosed with AMI was $84.9 \pm 10.4$ years (Table 1). Women accounted for $61.41 \%(\mathrm{n}=8347)$ of these patients. Among 1954 (14.38\%) patients who received invasive management procedures, $14.03 \%(\mathrm{n}=1907)$ received PCI and only $0.50 \%(n=68)$ received CABG. Most patients were diagnosed as non-STEMI ( $\mathrm{n}=11,793$, $86.76 \%$ ), and the type of dementia disorder for most patients was Alzheimer's disease $(\mathrm{n}=8308,61.12 \%)$, followed by other dementia $(n=3907,29.05 \%)$. The most common comorbidities were heart failure (78.45\%), cerebrovascular diseases (76.47\%) and diabetes mellitus (59.98\%). The most frequently prescribed drugs were anxiolytics (92.90\%), hypnotics and sedatives (85.90\%) and vasodilators $(85.16 \%)$. Overall, the median comorbidity score was $3(\mathrm{IQR}=2)$, and the median number of prescribed medications was $7(\mathrm{IQR}=3)$. Over half of the patients received home-visit treatment $(n=6960,51.20 \%)$. As for the use of long-term care within 3 months of AMI admission, $12.82 \%$ did not use long-term care services, $38.80 \%$ used home-visit care, $32.49 \%$ used residential facility care and $15.88 \%$ used outpatient facility care.

\section{Stratified Univariate Analyses}

A higher percentage of men than women underwent invasive procedures $(18.13 \%$ vs $12.02 \%$, Table S2). Men had a higher risk of DPC admission. The distribution of comorbidity score varied significantly by sex. Compared with men, women were prescribed more statins, calcium channel blockers, vasodilators, diuretics and lipid-lowering drugs, and women had significantly higher utilisation of homevisit treatment (women: $52.21 \%$ vs men: $49.60 \%$ ) and longterm care (women: $90.86 \%$ vs men: $81.32 \%$ ).

Women had a higher frequency of STEMI (Table S3). Most invasive procedures were performed in patients with STEMI (60.49\%, Table S4). Patients with STEMI were prescribed more drugs compared with patients with nonSTEMI. Compared with patients without invasive treatments, patients undergoing invasive procedures had higher comorbidity scores, were prescribed more drugs, and used less home-visit treatment and less long-term care. Invasive procedures did not differ significantly by type of dementia disorder (Table S5). A relatively high proportion of patients with vascular dementia received residential facility care $(39.97 \%)$, whereas home-visit care was more frequent among patients with Alzheimer's disease $(39.54 \%)$ or mixed dementia $(42.94 \%)$. Patients who used home-visit care or outpatient facility care showed 
Table I Baseline Characteristics of the Patients

\begin{tabular}{|c|c|}
\hline Characteristics & Values \\
\hline Age at $\mathrm{AMI}$, mean $\pm \mathrm{SD}$ & $84.9 \pm 10.4$ \\
\hline \multicolumn{2}{|l|}{ Age category (\%) } \\
\hline $65-74$ & $306(2.25)$ \\
\hline $75-84$ & $5164(37.99)$ \\
\hline $85-94$ & $6901(50.77)$ \\
\hline$\geq 95$ & I 222 (8.99) \\
\hline \multicolumn{2}{|l|}{ Sex (\%) } \\
\hline Men & $5246(38.59)$ \\
\hline Women & $8347(6 I .4 I)$ \\
\hline \multicolumn{2}{|l|}{ Type of AMI (\%) } \\
\hline STEMI & $1800(13.24)$ \\
\hline Non-STEMI & II,793 (86.76) \\
\hline Invasive management (\%) & $1954(14.38)$ \\
\hline $\mathrm{PCl}$ & $1907(14.03)$ \\
\hline CABG & $68(0.50)$ \\
\hline \multicolumn{2}{|l|}{ Type of dementia disorder (\%) } \\
\hline Alzheimer's disease & $8308(61.12)$ \\
\hline Vascular dementia & $628(4.62)$ \\
\hline Mixed dementia & $708(5.21)$ \\
\hline Other dementias & $3949(29.05)$ \\
\hline DPC (\%) & $3844(28.28)$ \\
\hline \multicolumn{2}{|l|}{ Comorbidities (\%) } \\
\hline Shock & $4 \mid 43(30.48)$ \\
\hline Diabetes mellitus & $8153(59.98)$ \\
\hline Heart failure & $10,664(78.45)$ \\
\hline Cardiac dysrhythmia & $712(5.24)$ \\
\hline Cerebrovascular disease & $10,395(76.47)$ \\
\hline Pulmonary edema & $430(3.16)$ \\
\hline Acute renal disease & $2817(20.72)$ \\
\hline Chronic renal disease & $3126(23.00)$ \\
\hline Cancer & $3948(29.04)$ \\
\hline Comorbidity score, median (IQR) & $3(2)$ \\
\hline \multicolumn{2}{|l|}{ Drugs (\%) } \\
\hline Antithrombotic & $9425(69.34)$ \\
\hline Statins & $612 \mid(45.03)$ \\
\hline Beta blockers & $3510(25.82)$ \\
\hline Calcium channel blockers & $7633(56.15)$ \\
\hline ACE-I/ARB & $4522(33.27)$ \\
\hline Vasodilators & II,576 (85.16) \\
\hline Diuretics & $9637(70.90)$ \\
\hline Antidiabetic & $3260(23.98)$ \\
\hline Lipid-lowering drugs & $6403(47.11)$ \\
\hline Cholinesterase inhibitors & $2656(19.54)$ \\
\hline Antipsychotic & $10,438(76.79)$ \\
\hline Anxiolytics & $12,628(92.90)$ \\
\hline Hypnotics and sedatives & II,676 (85.90) \\
\hline
\end{tabular}

(Continued)
Table I (Continued).

\begin{tabular}{|l|c|}
\hline Characteristics & Values \\
\hline Total number of drugs, median (IQR) & $7(3)$ \\
\hline Home-visit treatment (\%) & $6960(51.20)$ \\
\hline Type of long-term care (\%) & $1743(12.82)$ \\
No long-term care & $5274(38.80)$ \\
Home-visit care & $4417(32.49)$ \\
Residential facility care & $2159(15.88)$ \\
Outpatient facility care & \\
\hline
\end{tabular}

Abbreviations: $\mathrm{PCl}$, percutaneous coronary interventions; $\mathrm{CABG}$, coronary artery bypass grafting; AMI, acute myocardial infarction; STEMI, ST-elevation myocardial infarction; ACE-l, angiotensin-converting enzyme inhibitor; ARB, angiotensin receptor blocker; DPC, diagnosis procedure combination.

higher proportions of home-visit treatment after AMI (home-visit care: $56.54 \%$, outpatient facility care: $66.19 \%$, Table S6).

\section{Multilevel Logistic Models}

Multilevel logistic models were used to estimate the effect of the use of invasive management (Model 1) and longterm care (Model 2, Table 2). Model 1 shows that patients aged over 85 years had significantly lower use in invasive management, compared with other age groups (85-94 years: $\mathrm{OR}=0.62,95 \% \mathrm{CI}: 0.43-0.90 ; \geq 95$ years: $\mathrm{OR}=$ $0.40,95 \%$ CI: $0.26-0.63)$. Women were less likely than men to undergo invasive procedures during hospitalisation for AMI (OR $=0.65,95 \%$ CI: $0.58-0.74)$. A significantly higher use of invasive procedures was observed among patients with higher numbers of drugs (OR $=1.63,95 \%$ CI: 1.58-1.69) and among those with DPC admission (OR $=9.91,95 \%$ CI: 8.77-11.20). Significant variation was observed in the SMA level $\left(\delta^{2}\right.$ 0.068). Random effect estimates suggested significant between-SMA variability $(\mathrm{ICC}=0.020, \mathrm{MOR}=1.627)$.

In Model 2, patients who underwent invasive procedures show a lower odds of using long-term care $(\mathrm{OR}=$ $0.68,95 \%$ CI: $0.57-0.80)$. The odds of long-term care use increased with age category ( $75-84$ years: $\mathrm{OR}=1.77,95 \%$ CI: $1.35-2.32$; $85-94$ years: OR $=4.00,95 \%$ CI: $3.04-$ 5.28 ; $\geq 95$ years: $\mathrm{OR}=7.35,95 \%$ CI: $5.03-10.72$ ). Significant associations with a higher proportion of longterm care use were observed for women $(\mathrm{OR}=1.92,95 \%$ CI: 1.72-2.14) and patients receiving home-visit treatment $(\mathrm{OR}=3.87,95 \%$ CI: 3.43-4.37). The use of invasive management procedures was shown to be significantly higher among patients with a higher total number of 
Table 2 Multilevel Logistic Models Predicting the Use of Invasive Procedures and Long-Term Care

\begin{tabular}{|c|c|c|c|c|}
\hline & \multicolumn{2}{|c|}{ Model I } & \multicolumn{2}{|c|}{ Model 2} \\
\hline & OR & $95 \% \mathrm{Cl}$ & OR & $95 \% \mathrm{Cl}$ \\
\hline \multicolumn{5}{|l|}{ Fixed effects } \\
\hline \multicolumn{5}{|l|}{ Age category } \\
\hline $65-74$ & Ref. & & & \\
\hline $75-84$ & 0.76 & $0.53-1.10$ & 1.77 & $1.35-2.32$ \\
\hline $85-94$ & 0.62 & $0.43-0.90$ & 4.00 & $3.04-5.28$ \\
\hline$\geq 95$ & 0.40 & $0.26-0.63$ & 7.35 & $5.03-10.7$ \\
\hline \multicolumn{5}{|l|}{ Sex } \\
\hline Men & Ref. & & Ref. & \\
\hline Women & 0.65 & $0.58-0.74$ & 1.92 & $1.72-2.14$ \\
\hline Number of comorbidities & 0.96 & $0.92-1.00$ & 1.06 & $1.01-1.10$ \\
\hline Total number of drugs & 1.63 & $1.58-1.69$ & 1.05 & $1.02-1.07$ \\
\hline DPC & 9.91 & $8.77-11.2$ & 1.01 & $0.90-1.17$ \\
\hline Invasive procedures & & & 0.68 & $0.57-0.80$ \\
\hline Home-visit treatment & & & 3.87 & $3.43-4.37$ \\
\hline \multicolumn{5}{|l|}{ Random effects } \\
\hline$\delta^{2}$ (SMA level) & & 0.068 & & 0.036 \\
\hline ICC (SMA level) & & 0.020 & & 0.011 \\
\hline MOR (SMA level) & & 1.627 & & 1.516 \\
\hline
\end{tabular}

Notes: Model I is a multilevel logistic model predicting the use of invasive procedures. Age category, sex, number of comorbidities, total number of drugs, and DPC status were included as predictors in this model. The outcome was the use of invasive management procedures. Model 2 is a multilevel logistic model predicting the use of long-term care. Age category, sex, number of comorbidities, total number of drugs, DPC status, invasive management, and home-visit treatment were included as predictors in this model. The outcome was the use of long-term care.

Abbreviations: OR, odds ratio; $\mathrm{Cl}$, confidence interval; ICC, intraclass correlation coefficient; DPC, diagnosis procedure combination; SMA, secondary medical area.

medications $(\mathrm{OR}=1.69,95 \% \mathrm{CI}: 1.64-1.73) . \mathrm{DPC}$ status was not significantly associated with the use of long-term care. Random effect estimates suggested significant between-SMA variability $(\mathrm{ICC}=0.011, \mathrm{MOR}=1.516)$.

\section{Multilevel Survival Models}

Significantly higher hazards of death were observed as age category increased in Model 3 (Table 3). Women had a lower hazard of death relative to men $(\mathrm{HR}=0.71$, 95\% CI: 0.68-0.74). Patients who underwent invasive management procedures had a lower hazard of death than those who did not $(\mathrm{HR}=0.71,95 \% \mathrm{CI}$ : 0.66-0.77). Patients who received long-term care had a significantly lower hazard of death relative to patients without longterm care (home-visit care: $\mathrm{HR}=0.89$, 95\% CI: $0.83-$
0.96 ; residential facility care: $\mathrm{HR}=0.81,95 \% \mathrm{CI}: 0.75-$ 0.87 ; outpatient facility care: $\mathrm{HR}=0.73,95 \% \mathrm{CI}$ : $0.67-$ $0.80)$. Marginal survivor functions for invasive management and long-term care are plotted in Figure 1. DPC admission was associated with decreased hazard ratios. Whereas the hazard of death was significantly higher with larger numbers of comorbidities ( $\mathrm{HR}=1.13,95 \%$ CI: 1.12-1.15), a lower hazard was observed with larger numbers of medications ( $\mathrm{HR}=0.91,95 \% \mathrm{CI}$ : 0.91-0.92). Between-SMA variability no longer existed after the introduction of SMA-level characteristics. Similar betweenindividual-level outcomes were observed in Model 4, and significant hazards of death were not observed for any SMA-level characteristics.

\section{Discussion}

We used prefecture-wide medical insurance and long-term care insurance databases to examine management strategies and survival outcomes for AMI in older patients with dementia over a 6-year period. Overall, 14.38\% of these patients underwent invasive procedures, and $87.18 \%$ received long-term care after AMI. Invasive procedures were commonly performed for patients with STEMI $(60.49 \%)$ and those with DPC admission (75.84\%). Patients who underwent invasive procedures used homevisit care relatively frequently $(43.09 \%)$. Patients aged $\geq$ 85 years and women were less likely than others to undergo invasive procedures. Women and patients who received invasive procedures and long-term care had relatively low risks of death.

The presence of vascular atherosclerotic disease is typical among patients with dementia, ${ }^{31}$ and older adults with dementia are actually at a higher risk of AMI. ${ }^{32}$ However, in our study, the proportion of patients with dementia with AMI diagnosis was only $6.32 \%$, which was comparatively lower than expected. It is likely that AMI cases were under-diagnosed in older adults with dementia due to the atypical presentation AMI syndrome. ${ }^{33,34}$ There are also communication difficulties for patients with dementia to accurately describe their symptoms. The estimation of number of AMI cases among dementia patients is not, however, the main objective of the study as we were more interested in finding out the influence of management strategies for these older adults that could be potentially useful in improving of quality of life. As some previous studies have demonstrated that long-term care can improve the quality of life for older adults, ${ }^{35-37}$ it is anticipated that both survival 
Table 3 Multilevel Survival Models Predicting the Survival Outcomes of the Use of Invasive Procedures and Long-Term Care

\begin{tabular}{|c|c|c|c|c|}
\hline & \multicolumn{2}{|c|}{ Model 3} & \multicolumn{2}{|c|}{ Model 4} \\
\hline & HR & $95 \% \mathrm{Cl}$ & HR & $95 \% \mathrm{Cl}$ \\
\hline \multicolumn{5}{|l|}{ Fixed effects } \\
\hline \multicolumn{5}{|l|}{ Between-individual } \\
\hline \multicolumn{5}{|l|}{ Age category } \\
\hline $65-74$ & Ref. & & Ref. & \\
\hline $75-84$ & 1.39 & $1.18-1.63$ & 1.39 & $1.19-1.64$ \\
\hline $85-94$ & 2.16 & $1.84-2.54$ & 2.17 & $1.85-2.55$ \\
\hline$\geq 95$ & 3.58 & $3.02-4.25$ & 3.59 & $3.03-4.26$ \\
\hline \multicolumn{5}{|l|}{ Sex } \\
\hline \multicolumn{5}{|l|}{ Men } \\
\hline Women & 0.71 & $0.68-0.74$ & 0.71 & $0.68-0.74$ \\
\hline Invasive procedures & 0.71 & $0.66-0.77$ & 0.71 & $0.66-0.77$ \\
\hline DPC & 0.94 & $0.89-0.99$ & 0.94 & $0.89-0.99$ \\
\hline Number of comorbidities & 1.13 & $1.12-1.15$ & 1.13 & $1.12-1.15$ \\
\hline Total number of drugs & 0.91 & $0.91-0.92$ & 0.92 & $0.91-0.92$ \\
\hline Home-visit treatment & 1.03 & $0.98-1.07$ & 1.03 & $0.99-1.08$ \\
\hline \multicolumn{5}{|l|}{ Type of long-term care } \\
\hline No long-term care & Ref. & & Ref. & \\
\hline Home-visit care & 0.89 & $0.83-0.96$ & 0.89 & $0.83-0.96$ \\
\hline Residential facility care & 0.81 & $0.75-0.87$ & 0.81 & $0.75-0.87$ \\
\hline Outpatient facility care & 0.73 & $0.67-0.80$ & 0.73 & $0.67-0.80$ \\
\hline \multicolumn{5}{|l|}{ Between-SMA } \\
\hline Population density & & & 0.99 & $0.99-1.00$ \\
\hline Psychiatrist density & & & 0.99 & $0.97-1.02$ \\
\hline Cardiologist density & & & 1.00 & $0.98-1.02$ \\
\hline Dementia rate & & & 1.01 & $0.90-1.17$ \\
\hline MI rate & & & 0.97 & $0.88-1.07$ \\
\hline Psychiatric bed density & & & 1.00 & $0.99-1.00$ \\
\hline Home-visit care hospital density & & & 1.00 & $0.92-1.08$ \\
\hline Home-visit care clinic density & & & 1.00 & $0.98-1.00$ \\
\hline Long-term care staff density & & & 1.00 & $0.99-1.01$ \\
\hline \multicolumn{5}{|l|}{ Random effects } \\
\hline Between-SMA variability & \multicolumn{2}{|c|}{$0.0004(0.0000-0.0656)$} & \multicolumn{2}{|c|}{-} \\
\hline
\end{tabular}

Notes: Model 3 is a multilevel survival model by including individual-level covariates. Model 4 is a multilevel survival model by including both individual-level and SMA-level covariates.

Abbreviations: HR, hazard ratio; Cl, confidence interval; DPC, diagnosis procedure combination; SMA, secondary medical area.

outcome and quality of life among AMI older adults with dementia could be enhanced by the adoption of proper treatment and post-care strategies.

Our finding that invasive procedure management strategies were mostly used among STEMI patients and in extreme emergency conditions (DPC) are in accordance with recent guidelines recommending an early invasive procedure as a treatment strategy for such patients. ${ }^{38,39}$ We also found that the type of dementia was not significantly associated with the utilisation of invasive procedures for older patients. However, the invasive procedure rate for AMI among patients with dementia was remarkably lower compared with previous research on PCI use. $^{40-42}$ The reasons for this low rate may include 

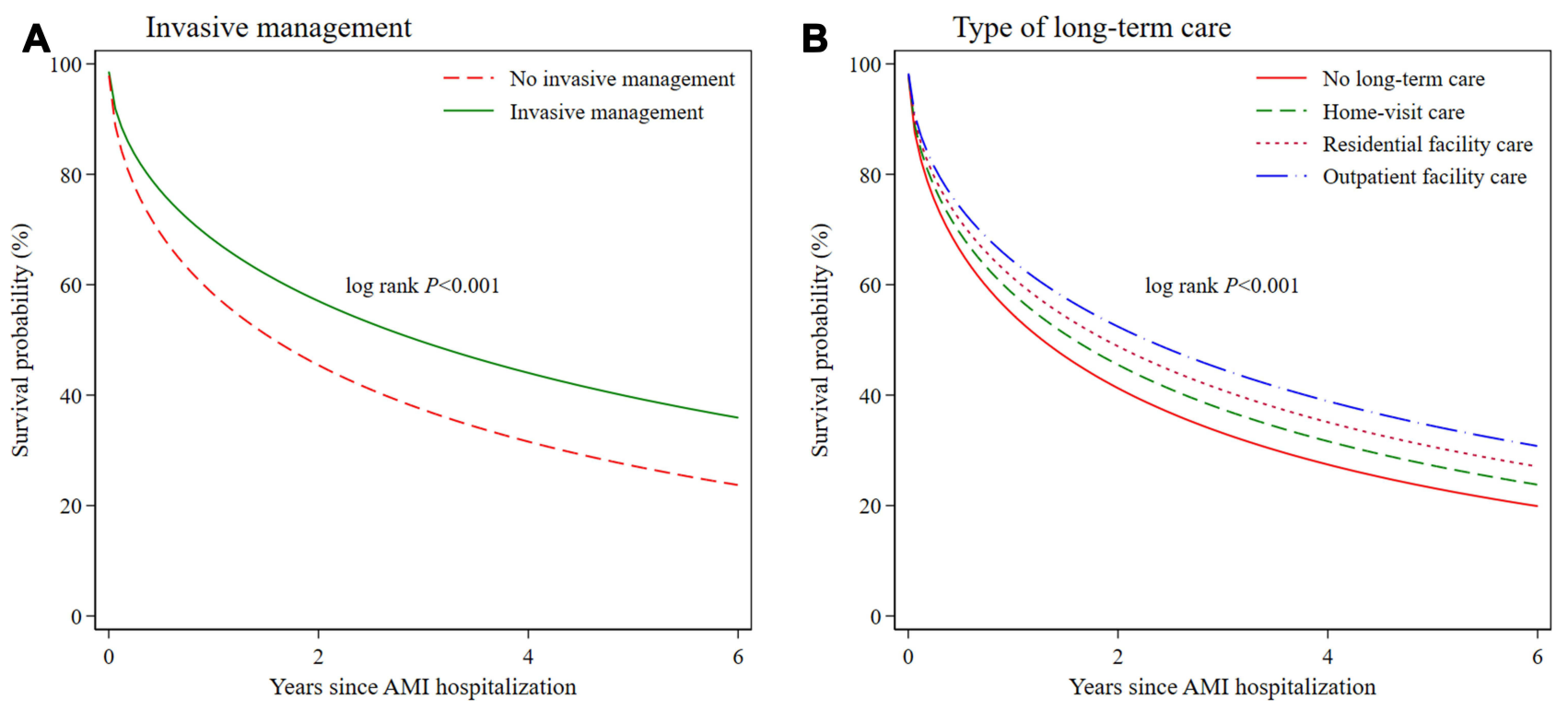

Figure I Marginal survivor function for the use of invasive management procedures (A) and long-term care (B).

complex comorbidities, uncertain benefits and insufficient guidelines, resulting in patients' conservative preferences for treatment and physicians' difficulty in making judgements and recommendations for these patients. Our findings showed that patients undergoing invasive procedures usually had fewer comorbidities but were prescribed more drugs, compared with patients who did not undergo these procedures. Antithrombotic agents, vasodilators, anxiolytics, hypnotics and sedatives were commonly used for invasively managed patients. Unlike other surgeries, PCI does not require a long recovery time, which may contribute to better clinical effectiveness and efficiency for older people. ${ }^{43,44}$ We also found that patients who underwent invasive procedures had a lower odd of using longterm care, suggesting that invasive management of AMI may be clinically effective and efficient for older adults with dementia. These findings provide limited support for a beneficial effect of the invasive management of AMI.

Our study is consistent with a previous study demonstrating that AMI patients with dementia had better post-discharge survival outcomes when invasive procedures were used. ${ }^{45}$ In line with other previous studies, our findings indicated that women less frequently underwent invasive procedures, more frequently used long-term care and consequently had a better survival rate. ${ }^{46,47}$ In contrast, the oldest old patients less frequently underwent invasive procedures for management and had a higher mortality risk, possibly because of complex comorbidities in this group. ${ }^{48}$ In our findings, although significant SMA-level variability was not observed and SMAlevel characteristics had no significant effect on survival outcome, patients who received invasive procedures and longterm care had a relatively low mortality risk, regardless of the specific type of services. Taken together, these findings suggest that long-term care after AMI may play an important role in reducing mortality risk, in addition to invasive procedure management. Therefore, long-term care combined with invasive procedures may provide a promising management strategy for AMI among patients with dementia.

The study has several limitations. First, we obtained insurance claims data that were not specifically designed for clinical research. Some clinical information was unavailable. Thus, confounding factors such as the severity of comorbid conditions, might not be adequately examined. In this study, we only assigned 1 point to the predetermined comorbidity whenever a record exists in the database. Further studies examining the applicability of our method in assigning points for AMI comorbidity among older patients with dementia are warranted. In addition, the SMA-level variables were calculated from population census surveys, which are not all conducted every year. Therefore, we included SMA-level variables from the 2014 2015 period. Despite these limitations, to our knowledge, our study is among the first to examine management strategies and outcomes in AMI patients with dementia by combining medical treatment and long-term care in SMAs. Using a 6-year prefecture-level dataset allowed us to better assess the outcomes. Additionally, the databases we used had a high penetration rate, covering more than 600,000 insured people 
annually; this ensured wide population coverage and a large sample size.

\section{Acknowledgments}

The authors thank the Wide-area Association of Latter Stage Elderly Healthcare of Fukuoka Prefecture for their provision of a health care claims database. We thank Jennifer Barrett, $\mathrm{PhD}$, from Edanz (https://jp.edanz.com/ac) for editing a draft of this manuscript.

\section{Funding}

This research did not receive any specific grant from funding agencies in the public, commercial or not-forprofit sectors.

\section{Disclosure}

The authors declare no potential conflicts of interest.

\section{References}

1. Briffa T, Hickling S, Knuiman M, et al. Long term survival after evidence based treatment of acute myocardial infarction and revascularisation: follow-up of population based Perth MONICA cohort, 1984-2005. BMJ. 2009;338:b36. doi:10.1136/bmj.b36

2. Asaria P, Elliott P, Douglass M, et al. Acute myocardial infarction hospital admissions and deaths in England: a national follow-back and follow-forward record-linkage study. Lancet Public Health. 2017;2(4):e191-e201. doi:10.1016/s2468-2667(17)30032-4

3. Livingston G, Huntley J, Sommerlad A, et al. Dementia prevention, intervention, and care: 2020 report of the Lancet Commission. Lancet. 2020;396(10248):413-446. doi:10.1016/s0140-6736(20)30367-6

4. Thiele H, Akin I, Sandri M, et al. PCI Strategies in Patients with Acute Myocardial Infarction and Cardiogenic Shock. $N$ Engl J Med. 2017;377(25):2419-2432. doi:10.1056/NEJMoa1710261

5. Fox KA, Poole-Wilson PA, Henderson RA, et al. Interventional versus conservative treatment for patients with unstable angina or non-ST-elevation myocardial infarction: the British Heart Foundation RITA 3 randomised trial. Randomized Intervention Trial of unstable Angina. Lancet. 2002;360(9335):743-751. doi:10.1016/s01406736(02)09894-x

6. Ryan TJ. Revascularization for acute myocardial infarction. Strategies in need of revision. Circulation. 1990;82(3 Suppl):Ii110116.

7. Phan DQ, Rostomian AH, Schweis F, et al. Revascularization versus medical therapy in patients aged 80 years and older with acute myocardial infarction. J Am Geriatr Soc. 2020;68(11):2525-2533. doi:10.1111/jgs. 16747

8. Tehrani DM, Darki L, Erande A, Malik S. In-hospital mortality and coronary procedure use for individuals with dementia with acute myocardial infarction in the United States. $J$ Am Geriatr Soc. 2013;61(11):1932-1936. doi:10.1111/jgs.12497

9. George J, Long S, Vincent C. How can we keep patients with dementia safe in our acute hospitals? A review of challenges and solutions. $J$ R Soc Med. 2013;106(9):355-361. doi:10.1177/ 0141076813476497

10. Catic AG, Mattison ML, Bakaev I, Morgan M, Monti SM, Lipsitz L. ECHO-AGE: an innovative model of geriatric care for long-term care residents with dementia and behavioral issues. J Am Med Dir Assoc. 2014;15(12):938-942. doi:10.1016/j.jamda.2014.08.014
11. Sivananthan SN, McGrail KM. Diagnosis and disruption: population-level analysis identifying points of care at which transitions are highest for people with dementia and factors that contribute to them. J Am Geriatr Soc. 2016;64(3):569-577. doi:10.1111/ jgs. 14033

12. Muraki I, Yamagishi K, Ito Y, et al. Caregiver burden for impaired elderly Japanese with prevalent stroke and dementia under long-term care insurance system. Cerebrovasc Dis. 2008;25(3):234-240. doi: $10.1159 / 000113861$

13. Kingston A, Comas-Herrera A, Jagger C. Forecasting the care needs of the older population in England over the next 20 years: estimates from the Population Ageing and Care Simulation (PACSim) modelling study. Lancet Public Health. 2018;3(9):e447-e455. doi:10.1016/ s2468-2667(18)30118-x

14. Tsutsui T, Muramatsu N. Care-needs certification in the long-term care insurance system of Japan. J Am Geriatr Soc. 2005;53 (3):522-527. doi:10.1111/j.1532-5415.2005.53175.x

15. Tomita N, Yoshimura K, Ikegami N. Impact of home and community-based services on hospitalisation and institutionalisation among individuals eligible for long-term care insurance in Japan. BMC Health Serv Res. 2010;10:345. doi:10.1186/1472-6963-10-345

16. Sakamoto HRM, Nomura S, Okamoto E, et al. Japan Health System Review. Vol. 8. New Delhi: World Health Organization, Regional Office for South-East Asia; 2018.

17. Saunderson CE, Brogan RA, Simms AD, Sutton G, Batin PD, Gale CP. Acute coronary syndrome management in older adults: guidelines, temporal changes and challenges. Age Ageing. 2014;43 (4):450-455. doi:10.1093/ageing/afu034

18. Chanti-Ketterl M, Pathak EB, Andel R, Mortimer JA. Dementia: a barrier to receiving percutaneous coronary intervention for elderly patients with ST-elevated myocardial infarction. Int $J$ Geriatr Psychiatry. 2014;29(9):906-914. doi:10.1002/gps.4078

19. So L, Evans D, Fau-Quan H, Quan H. ICD-10 coding algorithms for defining comorbidities of acute myocardial infarction. BMC Health Serv Res. 2006;6:161. doi:10.1186/1472-6963-6-161

20. Yamada M, Arai H. Long-term care system in Japan. Ann Geriatr Med Res. 2020;24(3):174-180. doi:10.4235/agmr.20.0037

21. Ito $\mathrm{T}$, Mori $\mathrm{T}$, Takahashi $\mathrm{H}$, et al. Prevention services via public long-term care insurance can be effective among a specific group of older adults in Japan. BMC Health Serv Res. 2021;21(1):531. doi:10.1186/s12913-021-06495-0

22. Li Y, Babazono A, Jamal A, Jiang P, Fujita T. Cost-Sharing Effects on Hospital Service Utilization Among Older People in Fukuoka Prefecture, Japan. Int J Health Policy Manag. 2020. doi:10.34172/ ijhpm. 2020.190

23. Nojiri S, Itoh H, Kasai T, et al. Comorbidity status in hospitalized elderly in Japan: analysis from National Database of Health Insurance Claims and Specific Health Checkups. Sci Rep. 2019;9 (1):20237. doi:10.1038/s41598-019-56534-4

24. Kimura T, Sugitani T, Nishimura T, Ito M. Validation and recalibration of Charlson and elixhauser comorbidity indices based on data from a Japanese insurance claims database. Jpn J Pharmacoepidemiol. 2019;24(2):53-64. doi:10.3820/jjpe.24.e2

25. Kimura S, Sato T, Ikeda S, Noda M, Nakayama T. Development of a database of health insurance claims: standardization of disease classifications and anonymous record linkage. J Epidemiol. 2010;20 (5):413-419. doi:10.2188/jea.je20090066

26. Gelman A. Multilevel (Hierarchical) Modeling: what It Can and Cannot Do. Technometrics. 2006;48(3):432-435. doi:10.1198/ 004017005000000661

27. Merlo J, Chaix B, Ohlsson H, et al. A brief conceptual tutorial of multilevel analysis in social epidemiology: using measures of clustering in multilevel logistic regression to investigate contextual phenomena. J Epidemiol Community Health. 2006;60(4):290-297. doi:10.1136/jech.2004.029454 
28. Austin PC, Merlo J. Intermediate and advanced topics in multilevel logistic regression analysis. Stat Med. 2017;36(20):3257-3277. doi:10.1002/sim.7336

29. Larsen K, Merlo J. Appropriate assessment of neighborhood effects on individual health: integrating random and fixed effects in multilevel logistic regression. Am J Epidemiol. 2005;161(1):81-88. doi:10.1093/aje/kwi017

30. Lowder EM, Amlung J, Ray BR. Individual and county-level variation in outcomes following non-fatal opioid-involved overdose. J Epidemiol Community Health. 2020;74(4):369-376. doi:10.1136/ jech-2019-212915

31. Maloberti A, Fribbi F, Motto E, et al. Ankle-brachial index is a predictor of in-hospital functional status but not of complications in hospitalized elderly patients. Gerontology. 2021:1-7. doi:10.1159/ 000514450

32. Justin BN, Turek M, Hakim AM. Heart disease as a risk factor for dementia. Clin Epidemiol. 2013;5:135-145. doi:10.2147/CLEP. S30621

33. DeVon HA, Mirzaei S, Zègre-Hemsey J. Typical and atypical symptoms of acute coronary syndrome: time to retire the terms? $\mathrm{J} \mathrm{Am}$ Heart Assoc. 2020;9(7):e015539. doi:10.1161/jaha.119.015539

34. Banovic S, Zunic LJ, Sinanovic O. Communication difficulties as a result of dementia. Mater Sociomed. 2018;30(3):221-224. doi: $10.5455 / \mathrm{msm} .2018 .30 .221-224$

35. Lawrence V, Fossey J, Ballard C, Moniz-Cook E, Murray J. Improving quality of life for people with dementia in care homes: making psychosocial interventions work. Br J Psychiatry. 2012;201 (5):344-351. doi:10.1192/bjp.bp.111.101402

36. Sloane PD, Zimmerman S, Williams CS, Reed PS, Gill KS, Preisser JS. Evaluating the quality of life of long-term care residents with Dementia. Gerontologist. 2005;1(1):37-49. doi:10.1093/geront/ 45.suppl_1.37

37. Kane RA. Long-term care and a good quality of life: bringing them closer together. Gerontologist. 2001;41(3):293-304. doi:10.1093/geront $/ 41.3 .293$

38. Kimura K, Kimura T, Ishihara M, et al. JCS 2018 guideline on diagnosis and treatment of acute coronary syndrome. Circ J. 2019;83(5):1085-1196. doi:10.1253/circj.CJ-19-0133

39. Ozaki Y, Katagiri Y, Onuma Y, et al. CVIT expert consensus document on primary percutaneous coronary intervention (PCI) for acute myocardial infarction (AMI) in 2018. Cardiovasc Interv Ther. 2018;33(2):178-203. doi:10.1007/s12928-018-0516-y
40. Randall DA, Jorm LR, Lujic S, O’Loughlin AJ, Eades SJ, Leyland AH. Disparities in revascularization rates after acute myocardial infarction between aboriginal and non-aboriginal people in Australia. Circulation. 2013;127(7):811-819. doi:10.1161/ circulationaha.112.000566

41. Berlin C, Jüni P, Endrich O, Zwahlen M. Revascularization Treatment of Emergency Patients with Acute ST-Segment Elevation Myocardial Infarction in Switzerland: results from a Nationwide, Cross-Sectional Study in Switzerland for 2010-2011. PLoS One. 2016;11(4):e0153326. doi:10.1371/journal.pone.0153326

42. Li Y, Babazono A, Jamal A, Fujita T, Yoshida S, Kim S-A. Variation in the use of percutaneous coronary interventions among older patients with acute coronary syndromes: a multilevel study in Fukuoka, Japan. Int J Equity Health. 2021;20(1):80. doi:10.1186/ s12939-021-01415-4

43. Costa-Font J, Jimenez-Martin S, Vilaplana C. Does long-term care subsidization reduce hospital admissions and utilization? J Health Econ. 2018;58:43-66. doi:10.1016/j.jhealeco.2018.01.002

44. Hamada S, Takahashi $\mathrm{H}$, Sakata $\mathrm{N}$, et al. Household Income Relationship With Health Services Utilization and Healthcare Expenditures in People Aged 75 Years or Older in Japan: a Population-Based Study Using Medical and Long-term Care Insurance Claims Data. J Epidemiol. 2019;29(10):377-383. doi:10.2188/jea.JE20180055

45. Cermakova P, Szummer K, Johnell K, et al. Management of acute myocardial infarction in patients with dementia: data from SveDem, the Swedish dementia registry. J Am Med Dir Assoc. 2017;18 (1):19-23. doi:10.1016/j.jamda.2016.07.026

46. Anand SS, Xie CC, Mehta S, et al. Differences in the management and prognosis of women and men who suffer from acute coronary syndromes. J Am Coll Cardiol. 2005;46(10):1845-1851. doi:10.1016/ j.jacc.2005.05.091

47. Mehta LS, Beckie TM, DeVon HA, et al. Acute myocardial infarction in women: a scientific statement from the American heart association. Circulation. 2016;133(9):916-947. doi:10.1161/cir.00000000000 00351

48. Girerd N, Magne J, Rabilloud M, et al. The impact of complete revascularization on long-term survival is strongly dependent on age. Ann Thorac Surg. 2012;94(4):1166-1172. doi:10.1016/j. athoracsur.2012.05.023
Clinical Epidemiology

\section{Publish your work in this journal}

Clinical Epidemiology is an international, peer-reviewed, open access, online journal focusing on disease and drug epidemiology, identification of risk factors and screening procedures to develop optimal preventative initiatives and programs. Specific topics include: diagnosis, prognosis, treatment, screening, prevention, risk factor modification, systematic reviews, risk \& safety of medical interventions, epidemiology \& biostatistical methods, and evaluation of guidelines, translational medicine, health policies \& economic evaluations. The manuscript management system is completely online and includes a very quick and fair peer-review system, which is all easy to use.

\section{Dovepress}

